\title{
MAGNETIC FIELD GENERATION IN PROTOGALACTIC HALOS
}

\author{
Ralph E. Pudritz \\ Dept. of Physics, McMaster University, Hamilton, ON L8S 4M1
}

\section{Introduction}

It has become quite clear that dynamically significant magnetic fields of order $10^{-6}$ Gauss are present in galactic halos (Perry, these Proceedings) at redshifts up to $z \geq 2$. Comparable fields have been detected on cluster and even on supercluster scales (Giovannini, these Proceedings). The existence of such high fields is surprising because it is highly unlikely that primeval magnetic fields could have been much stronger than $10^{-18}$ Gauss. These observations require that there be an epoch of very vigorous dynamo action that can amplify this seed primordial field up to the observed values. Since strong fields are already in place by redshifts of $z \simeq 2$, this amplification process must operate very quickly. This argues against the idea that such fields could be generated on huge intercluster scales since the dynamical times are far too long. A much more promising site for the dynamo process is in the dark matter halos of young galaxies during the epoch of galaxy formation. The fundamental point is that vigorous dynamo action can take place in shocks that are expected to occur throughout the dark matter potential wells of protogalaxies (Pudritz and Silk 1989 (三PS), Pudritz 1989).

The generation of strong magnetic fields in protogalactic halos has a profound effect on the epoch of star formation in young galaxies. Shocks play a fundamental role in this process of field amplification. One of the strongest pieces of evidence in favour of the idea that shocks were prevalent during galaxy formation is that the luminous matter in galaxies is much more concentrated than the dark matter component. This implies that young galaxies were able to radiate away much of their gravitational binding energy (Silk 1983, Efstathiou and Silk 1983, Zel'dovich and Novikov 1983) presumably in radiative shocks. Thus oblique shocks must be ubiquitous throughout early dark matter halos. The second major feature of galaxy formation is that galactic angular momentum likely arises through tidal torques between neighbouring protogalactic fluctuations as they separate out from the Hubble expansion (Hoyle 1949, Peebles 1969). This means that subsequent star formation in protogalactic halos must overcome an angular momentum problem that is nearly as severe as that in the present epoch in galaxies. In our own interstellar medium, the evidence increasingly supports the idea that braking by hydromagnetic waves or disk winds can achieve this. Similarly, powerful dynamo-constructed fields in protogalactic halos likely play a fundamental role in solving the angular momentum problem of primordial stars. 
The presence of dynamically significant magnetic field at the time of primordial star formation makes a link between these two processes likely. The observations show that galactic disks may form later than the spheroids (Cowie 1988). The abundance of damped Ly $\alpha$ absorption line systems suggest that galactic disks form at $z \simeq 2$. These systems also are strongly magnetized (Wolfe 1988). The fact that the intergalactic medium at high redshifts $3 \leq z \leq 4$ is almost completely ionized (Gunn and Peterson 1965) argues for an epoch of massive star formation at that time that provided the ionizing radiation (Cowie 1988, Rees 1988). Dynamos produce fields exponentially quickly $B=B_{\text {seed }} \cdot \exp \left(t / t_{d y n}\right)$ so that the basic physics problem is to show that $t_{d y n}$ is short enough so that an initially weak seed magnetic field can be amplified to the observed values by a redshift of $z \geq 3$ in protogalactic halos. I briefly summarize a model of galaxy formation which naturally achieves this (for details see PS).

\section{Seed Fields}

Current inflationary theories of the early universe produce completely negligible primeval fields. Interesting seed fields can be manufactured using conventional gas dynamics in protogalaxies however. Mishustin and Ruzmaikin (1972) pointed out that Compton drag by the background radiation field on the gas in a rotating protogalaxy can generate a seed field. The drag is more severe for electrons than protons so that a tiny charge separation is produced, and therefore a tiny current which in turn generates a magnetic field. If galaxy formation takes place a $z_{f}=5$, a field of $B_{\text {seed }}=.83 \times 10^{-21} \mathrm{G}$ is produced. At redshifts $z_{f} \leq 100$ we find that the Biermann battery mechanism produces a somewhat stronger field of $B_{\text {seed }} \simeq 0.7 \times 10^{-18}$ Gauss. This mechanism also arises from the rotation of a protogalaxy and finally dominates because the background radiation field becomes ever more dilute.

The point is then that seed fields are incredibly small. Only exponential growth is going to produce the field that is measured in halos of galaxies. In order to estimate what $t_{d y n}$ ought to be, consider the following timing argument. One must go from a seed field of $10^{-19} \mathrm{G}$ to the observed $10^{-6} \mathrm{G}$ and take no more than several $10^{9}$ years- a protogalactic free fall time. These numbers are separated by 30 e-foldings, which implies that $t_{d y n} \leq 10^{8} \mathrm{yr}$. This is much less than the dynamical time of a protogalaxy and suggests that field generation must take place in many smaller regions. This same argument makes it highly unlikely that dynamos can produce significant fields on scales characterizing the IGM directly.

\section{Protogalaxy Model}

We suppose that primordial clouds of baryons fall into dark matter potential wells of mass $M_{\text {protgal }} \simeq 10^{12} \mathrm{M}_{\odot}$ characterizing a protogalaxy (White and Rees 1978). However, the standard cold dark matter picture of galaxy formation predicts a spectrum of lower mass dark matter halos, and it is possible gas infall could get started in objects with only $10 \%$ of the mass of these current bright galaxies (Carlberg and Couchman 1989). The temperature characterizing the gravitational potential well of galactic dark matter halos in the current epoch is $10^{6} \mathrm{~K}$ and typical infall speeds are $\simeq 100 \mathrm{~km} \mathrm{~s}^{-1}$. These halos have radii of order $100 \mathrm{kpc}$ and mean smoothed out gas densities of order $10^{-3} n_{-3} \mathrm{~cm}^{-3}$.

Primordial gas clouds will be disrupted during cloud-cloud collisions if the ram 
pressure in the shock exceeds the self gravity of the merged object. This implies that the surface density of merged objects exceeds $\mu_{c r i t}=8.5 n_{-3}^{1 / 2}\left(v_{s} / 100 \mathrm{kms}^{-1}\right)$ $\mathrm{M}_{\odot} \mathrm{pc}^{-2}$. This is large compared to the smoothed out baryonic surface density $\mu_{\text {bary }} \simeq 2.7 \mathrm{M}_{\odot} \mathrm{pc}^{-2}$.

There are two crucial properties of shocks that control the character of magnetic field generation. Dynamo action requires that gas possess vorticity. The fluctuations that arise in the early universe are irrotational, so that in general oblique shocks are mandatory for the first dynamos. Vorticity is generated because the shock jump conditions stipulate that while the tangential velocity component to the shock is continuous, the normal fluid velocity component decreases due to gas compression. Numerical simulations of uniform clouds undergoing off-centre collisions produce flattened bar like structures with associated vortical velocity fields (e.g. Lattanzio et al 1985).

The other important point is that these shock layers can undergo considerable cooling through the formation of molecular hydrogen. While gas of primordial composition ordinarily cannot cool much below $10^{4} \mathrm{~K}$ after recombination, in nonequilibrium conditions such as a shock, the formation of molecular hydrogen is possible. The reason is that a few more electrons are around so that the reactions $\mathrm{H}+e \rightarrow \mathrm{H}^{-} ; \mathrm{H}^{-}+\mathrm{H}=\mathrm{H}_{2}+e$ can proceed. Detailed calculations show that molecular fractions of $10^{-3}$ are produced under a wide variety of preshock densities and ionization backgrounds (MacLow and Shull 1986) and that the gas cools down to $100^{\circ} \mathrm{K}$.

Cooling is important because flattened layers produced in the shock become rather thin, allowing gravitational instability to develop. It is in these fragments that magnetic field generation and eventual star formation break out. The typical surface density of the shock layer is found by equating the heating rate in the shock to the $H_{2}$ cooling rate yielding $\mu_{c}=12.6 T_{2}^{-1} \mathrm{M}_{\odot} \mathrm{pc}^{-2}$ where the temperature is taken as $100 \mathrm{~K}$. This surface density is consistent with the requirement that a bound clump remain after the collision.

If it is supposed that primordial clouds undergoing collisions are uniform and isothermal then it is reasonable to assume that thin, uniform, self-gravitating, isothermal post shock layers are produced (see e.g. Lattanzio et al 1985). Infalling clouds may however be more like isothermal spheres, and the merged objects while showing a flattened bar, have centrally condensed regions. Nonetheless, as a first approximation, consider the former case. Then one can apply the standard theory of fragmentation in isothermal layers to deduce how massive the final chunks will be. One finds that the fiducial mass, radius, density, and rotation frequency of our $100^{\circ} \mathrm{K}$ fragments are $M_{c}=4.0 \times 10^{6} T_{2}^{3} \mathrm{M}_{\odot}, r_{c}=160 T_{2}^{2}$ pc., $n_{c}=2.2 \times 10^{4} T_{2}^{3}$ $\mathrm{cm}^{-3}$, and $\Omega_{c}=0.69 \times 10^{-8} T_{2}^{-3 / 2} \mathrm{yr}^{-1}$ respectively. These results are particularly interesting when compared with the Orion molecular cloud in our own interstellar medium which is forming massive stars. For Orion, $M_{c l} \geq 10^{5} \mathrm{M}_{\odot}, n \geq 10^{3} \mathrm{~cm}^{-3}$, and $T_{c l}=30-50^{\circ} \mathrm{K}$. The Jeans length in the primordial cloud is comparable to that in Orion now, in fact $M_{\text {Jeans, }}=2.3 M_{\text {Jeans, Ori }}$. Thus the first self gravitating star forming fragments in protogalaxies will form massive stars in this theory. This will happen if the angular momentum problem is solved by braking. The other important result here is that the dynamical time $\Omega_{c}^{-1}$ is of order $10^{8}$ years. We have in principle satisfied the requirements of the timing argument. 


\section{Dynamo Action in the Fragments}

Since the seed field is extraordinarily small, one can employ the dynamo equations in their kinematic form. Thus, since the vortical gas flow in the compressed fragments has nearly gravitational energy density, which is 24 orders of magnitude larger than $B_{\text {seed }}^{2} / 8 \pi$ the magnetic back reaction on the fluid is negligible. The dynamo in this layer is of $\alpha-\omega$ type, that is, shear in the fragment as well as rising, twisting motions of the fluid (the $\alpha$-effect) are responsible for tapping the gravitational energy in the gas and converting it into magnetic field. The process saturates when the magnetic field is of order the gravitational energy density, or $\simeq 10^{-6}$ Gauss. The $\alpha$ in these shocked layers arises due the the presence of a vertical density gradient in the isothermal layer, and the vorticity $\omega$ generated in the layer as a consequence of the oblique shock; ie, $\alpha \propto(\nabla \rho) . \omega$. The linearized dynamo equations show that there is a fastest growing mode of the field. The dynamo time scale is directly proportional to $\Omega^{-1}$, which is to be expected. A detailed calculation shows (PS) $t_{d y n}=6.0 \times 10^{7} T_{2}^{3 / 2}$ yrs so that the time required to generate a microgauss field in our fragments is $t_{\text {field }}=1.9 \times 10^{9} \mathrm{yr}$. The coherence length of this field is about $0.6 T_{2}^{2} \mathrm{kpc}$. This field however, aquires a much larger coherence length after massive star formation breaks out in the halo.

\section{The Protogalactic Halo and Beyond}

The $10^{6} \mathrm{M}_{\odot}$ clumps ultimately produce $\mathrm{OB}$ associations with up to $10^{3}-10^{4}$ $\mathrm{O}$ stars, supposing that the star formation efficiency is 10 percent efficient. Winds and supernovae from these earliest associations will carve superbubbles into the halo gas with radii as large as $5 \mathrm{kpc}$. Thus, within $10^{7}$ years of the formation of the first shocks, massive OB associations will turn on and continue to form over a dynamical time across the halo $\left(10^{9} \mathrm{yr}\right)$. The result is that the halo takes on a swiss cheese appearance wherein each shell has swept up a total of at least $10^{6} \mathrm{M}_{\odot}$. The gas in each of the shells is metal enriched due to the supernovae ejecta. In addition, the ionizing photons from the massive stars ionize the IGM. It is possible that globular cluster formation gets started in the subsequent generation of star formation.

When does massive star formation start? The simplest possibility is when the field is strong enough to magnetically brake star forming gas. Of course, gravitational torques can also transport angular momentum but it is not yet clear that they can do this job as completely, or as efficiently. Detailed calculations suggest that gravitational wave torques may be important only in the very innermost parts of protostellar disks so that even in this scenario magnetic braking of some kind is also required. With the magnetic braking conjecture, star formation commences at roughly $t \simeq t_{\text {field }}$, or at $z \simeq 3$ for a universe with critical density $\Omega=1$. This result implies that when the remaining gas in a halo finally collapses to form the protogalactic disk the associated magnetic field will be very strong. The field in the disk will be stronger than the halo by a factor $B_{\text {disk }} / B_{\text {halo }} \simeq\left(\rho_{\text {disk }} / \rho_{\text {halo }}\right)^{1 / 2}$. Thus, the fact that damped Ly $\alpha$ systems are amongst the most magnetized objects has a natural explanation. Note that star formation can proceed immediately in disks since they are very well magnetized at their birth.

The observation that strong magnetic field is associated with clusters of galaxies such as the Coma cluster finds a natural explanation in our theory. Since the dark matter halos of galaxies within a cluster basically form one large merged ob- 
ject, one expects that the magnetic fields produced by our mechanism (PS) will also fill the entire volume of the cluster. It is not necessary to suppose then that such fields were ejected out of their parent galaxies, or produced in the IGM well outside a galactic halo.

An exciting extension of the observed scale of magnetic fields in the universe was reported at this meeting; namely the detection of $326 \mathrm{MHz}$ radio emission that appears to connect the Coma cluster with the Abell 1367 cluster (Kim et al 1989). If this is synchotron emission, microgauss fields are implicated. The authors suggest that field generation outside of the body of galaxies is a possible explanation. Examples of such processes are amplification of weak seed fields in the turbulent wakes of galaxies or in the course of large scale cosmic explosions that generated the voids.

The observations can also be understood in the context of magnetic field generation within the $100 \mathrm{kpc}$ dark matter halos associated with galaxies. Large scale structure is demarcated by the collection of dark matter potential wells in cold dark matter cosmology. Thus magnetic field will appear to trace large scale structure. This must be so for bridges of galaxies between dominant clusters. Thus, any object that can be formed by merging a collection of dark matter halos, will be strongly magnetized by a redshift of $z \simeq 3$ in this theory (provided $\Omega=1$ ). This possibility can account for the presence of high RM for the Comma cluster because the individual galactic dark matter halos merge together to form a cluster halo. Similarly, each dark matter halo of galaxies that are strung out on a filament connecting two rich clusters has strong magnetic fields as discussed. This then produces an apparent magnetic bridge. If this conjecture is correct, then it will be possible to trace large scale structure of the universe by means of radio observations. One should not be too surprised if future $326 \mathrm{MHz}$ observations find that magnetic structure and the most luminous large scale structure do not follow one another precisely. According to current pictures of biased galaxy formation (Kaiser 1984), the most luminous galaxies form at the peaks in dark matter fluctuations and need not therefore follow the distribution of the bulk of the dark matter mass.

An important observational test of the theory presented here that magnetic field generation occurs as a consequence of galaxy formation would be to push back to redshifts of $z \simeq 3$. If galaxy formation gets started at $z \simeq 3$, then at even larger redshifts, one should begin to see a downturn in RM for $z>3$, since magnetic field generation may still be occurring at these larger redshifts. Since the quasars run out at red shifts exceeding four, one would have very few background quasars to test this with. If the protogalactic halo is put together at redshifts exceeding four, then one would be unlikely to observe any such effect using RM data or searches for synchotron emission. Potentially searches of this kind would be a very important addition to the procedures currently used to identify the epoch of galaxy formation.

I have enjoyed interesting conversations on these topics with Phil Kronberg and in particular, Joseph Silk. This work was supported by NSERC of Canada. 


\section{REFERENCES}

Carlberg, R.G., and Couchman, H.M.P. 1989, Ap. J., 340, 47.

Cowie, L.L 1988, in The Post Combination Universe, N. Kaiser and A.N. Lasenby eds. (Kluwer: Dordrecht), 1.

Efstathiou, G. and Silk, J. 1983, Fund. Cosmic Physics, 9, 1.

Gunn, J.E. and Peterson, B.A. 1965, Ap. J., 142, 1633.

Hoyle, F. 1949, in Problems of Cosmical Aerodynamics (Dayton, Ohio: Central Air Documents Office), 195.

Kaiser, N. 1984, Ap. J., 284, L9.

Kim, K.-T., Kronberg, P.P., Giovanninni, G., and Venture, T. 1989, Nature, 341, . Lattanzio J.C. et al 1985, M. N. R. A. S., 215, 125.

MacClow, M.M., and Shull, J.M. 1986, Ap. J., 302, 585.

Mishustin, I.N., and Ruzmaikin, A.A. 1972, Soviet Phys-JETP, 34, 233.

Peebles, P.J.E. 1969, Ap. J., 155, 393.

Pudritz, R.E. 1989, in Accretion Disks and Magnetic Fields in Astrophysics, G. Belvedere ed. (Kluwer: Dordrecht), 191.

Pudritz, R.E., and Silk, J. 1989, Ap. J., 342, 650.

Rees, M.J. 1988, in The Post Recombination Universe N. Kaiser and A.N. Lasenby eds. (Kluwer: Dordrecht), 101.

Silk, J. 1983, Nature, 301, 574.

White, S.D.M., and Rees, M.J. 1978, M. N. R. A. S., 183, 341.

Wolfe, A.M. 1988, in QSO Absorption Lines, J.C. Blades, D. Turnshek, and C.A. Norman eds. (Cambridge University Press: New York), 297.

Zel'dovich, Ya.B., and Novikov, I.D. 1983 in Relativistic Astrophysics II (Chicago: University of Chicago Press).

RUZMAIKIN: What can you say about the possibility of starting without galactic magnetic fields and creating it in a rotating protostellar cloud by dynamo action which makes the cloud contraction possible? [See a paper by Bisn.vaty-Kogan, Sunyaev and Ruzmaikin, 1973, Sov. Astron. J.]

PUDRITZ: Your suggestion is interesting. The theory we developed was based on the need to solve the angular momentum problem of star formation. In this view, magnetic field generation is first required before the conditions resembling a protostellar object could even be approached. The second point is that one also wants to explain the RM data for galactic halos. This might require dynamo scales that are much larger than that of individual protostellar regions.

PARKER: Could you say a few words on how you arrived at a unique value for the dynamo coefficient $\alpha$ ? What uncertainties are involved? Do we know enough about what happened in that early epoch to make any quantitative statement? Or do you mean to present your results only as a possibility?

PUDRITZ: The coefficient $\alpha$ was calculated by assuming that the vorticity $\underline{\omega}$ (generated in the oblique shock) and the vertical density gradient in 
the layer (assumed to be self-gravitating and isothermal) combine to give the pseudo-scalar $\alpha \propto \Delta \rho . \underline{\omega}$. We think we know the scale height if the gas cools by the $\mathrm{H}_{2}$ mechanism, and an estimate was made of $\underline{\omega}$ from numerical calculations. The scalings of $\alpha$ are not substantially different from your scalings for galactic disk dynamos (see Pudritz and Silk, 1989, Ap.J. 342, 650; and Pudritz, 1981, MNRAS 195, 897 for more details). The final uncertainty is in the stirred gas speed, which appears as the turbulent Mach number $M_{T}$ in the calculation. Fortunately, the growth rate does not strongly depend upon $M_{T}$.

MOUSCHOVIAS: Before your transparency showing the dynamo equations, you showed a long series of transparencies which confused me very much. I could not separate the assumptions from the conclusions. Have you actually solved any differential equations, or are you just giving qualitative or scaling arguments that shocks exist in the hot protogalactic medium, that the mass behind them is really characteristic of protogalaxies etc.? (And a note: That magnetic fields may be crucial for star formation, in that actual calculations have shown that they can resolve the angular momentum problem of star formation, is not a new point [see my article in this volume]; I have a long series of papers on magnetic braking [either alone or with Paleologou, and S. Morton] in the Astrophysical Journal from 1977 to 1985.)

PUDRITZ: Your second point - that magnetic braking solves the angular momentum problem and is not a new idea, is certainly true. In fact, I regard that physics as so well understood and worked upon that it is "safe" to extend it to primordial star formation. The new point is that no previous calculation of primordial star formation has considered the angular momentum issue. This is our contribution.

The analysis in our work is indeed based on a plethora of equations, and is not qualitative. The theory of self-gravitating layers is well worked out - we applied the results to our case. Vorticity generation is derived from published numerical calculations and checked with shock jump conditions. A full analysis of the dynamo equations also was derived. The input from cosmology is taken from recent ideas of galaxy formation (e.g. White and Rees, 1978, MNRAS; Baron and White, 1987, Ap.J.).

M.L. NORMAN: What is the spatial distribution of these halo shocks? Is there a single global shock surrounding the protogalaxy, or many small shocklets within it?

PUDRITZ: The shocks should be considered to fill the dark matter potential well. Their number depends upon the distribution function of the lower mass clumps $\left(10^{8}-10^{9} \mathrm{M}_{\odot}\right.$ ?) falling into our supposed $10^{12} \mathrm{M} \odot$ potential well. This latter function presumably can be calculated within the context of any particular dark matter cosmology, such as CDM (see for example Efstathiou and Rees, 1988, MNRAS). The picture we have painted is indeed one in which many shocklets are found throughout a halo and not of a grant "global" shock. 
MORRIS: Although you discussed primarily high-mass star formation, if you allow for low-mass stars, you would be describing the formation of a cluster of about the mass of a globular cluster. Are you tempted to consider the possibility of a prior enrichment process and then to identify your clusters with globular clusters?

PUDRITZ: The problem outlined is to account for the very first stars. This requires that the gas is as yet not enriched so that the only likely coolant is $\mathrm{H}_{2}$. While the mass of the first star-forming clouds is, as you noted, interesting, it is somewhat larger than globular cluster masses. Since massive-star formation is expected in our objects, I don't think that the remaining low-mass stars would form a bound association. To do the latter probably requires that massive-star formation be less prevalent in the basic cloud.

KULSRUD: With respect to the dynamo theory, you only need mean $\mathrm{B}^{2}$, not mean B over big scales. Can you get away with a smaller $\alpha$, since even $\alpha=0$ generates field albeit on a very small scale?

PUDRITZ: The linear instability analysis of the dynamo equations shows that the wave number for the fastest growing mode scales with $\alpha$ as $\mathrm{k}_{m} \propto \alpha^{1 / 3}$; while the growth time varies as $\mathrm{t}_{\mathrm{dyn}} \propto \alpha^{-2 / 3}$. As $\alpha$ decreases, the dominant mode goes to larger and larger scales, but it takes much longer to generate. In order to create significant field by $z \sim 3$ therefore, $\alpha$ cannot be too small.

KRONBERG: It is interesting, in connection with your speculation that galaxies amplified magnetic fields as they formed, that our magnetized bridge in the Coma-A1367 supercluster coincides quite well with the smoothed-out distribution of galaxies in the supercluster. 